\title{
Optical fibres contactless sensor for dynamic testing of lightweight structures
}

\author{
L. Bregant ${ }^{\mathrm{a}, *}$ and M. Bressanutti ${ }^{\mathrm{b}}$ \\ ${ }^{a}$ Dipartimento Ingegneria Meccanica, Università di Trieste Via Valerio 10, 34127 Trieste, Italy \\ ${ }^{\mathrm{b}}$ APE Research, Area Science Park Basovizza, Trieste, Italy
}

Received 2007

Revised 2007

\begin{abstract}
With dynamic testing, engineers describe activities focused on the identification of some properties of vibrating structures. This step requires for the measurements of excitations and responses signals, applying appropriate sensors directly on the test article. These instruments modify the system's mass and stiffness distributions and eventually the eigen-properties of the structure. These errors become unacceptable especially when testing lightweight structures. This paper shows the results of some tests performed on a small compressor with the purpose of identifying the blades' natural frequencies and modes. It compares the acquisitions performed with standard accelerometers and two different contact-less systems using as exciters either a micro-hammer or a micro inertial shaker. The paper shows how the contact-less sensors provide good quality data and consistent results in the mode identification phase.
\end{abstract}

Keywords: Contact-less sensors, dynamic testing, vibration, mass loading

\section{Introduction}

Very frequently test data are biased by different systematic errors due to the measurement chain and the adopted sensors. A common problem is related to the mass loading effect caused by the transducers' and exciters masses applied onto the structure. These, modify the system, yielding to wrong estimates of structural eigen-frequencies. The loading effect is particularly evident in multi-patch measurements where, due to the limitation of the acquisition system, sensors have to be moved along the structure. In these cases, each time the sensors are relocated, the mass distribution and thus the structure dynamic properties change. Eventually the impact of the mass loading effect can be different for the different runs and the consequent data inconsistency will make it more difficult to identify the modal parameters and to use the stabilization diagrams for the poles' selection [1,2]. The effect can be formalized with Eq. (1), where $Z_{0}$ is the original dynamic stiffness of the test object, $Z_{m}$ is the modified stiffness after the mass $\mathrm{m}_{k}$ addition:

$$
Z_{m}=Z_{0}-m_{k} \omega^{2}
$$

From Eq. (1) it's evident that the addition of the mass $\mathrm{m}_{k}$ reduces the dynamic stiffness of the structure and as a consequence, the extracted eigen-frequencies will shift to lower frequencies values. The mass loading effect becomes more important when the weight of the test structure is small and at higher frequencies values. A transducer placed on a nodal line will not cause mass loading effect while it will strongly influence the measured data if placed at anti-nodal locations [3]. The mass loading effect has been considered in the past, and several analytical corrective approaches have been proposed [4-6], but the usability of these methods is not always straightforward and their

*Corresponding author. E-mail: bregant@units.it. 


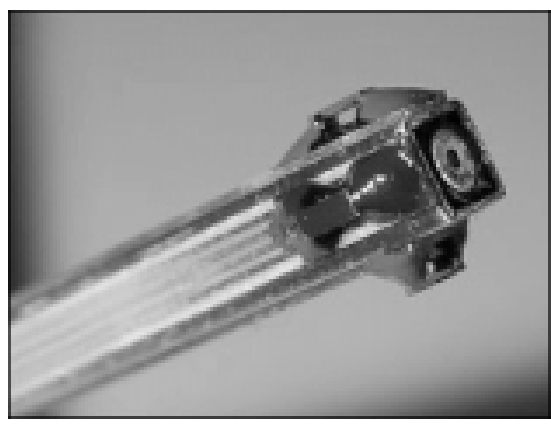

Fig. 1. Microflown USP probe.

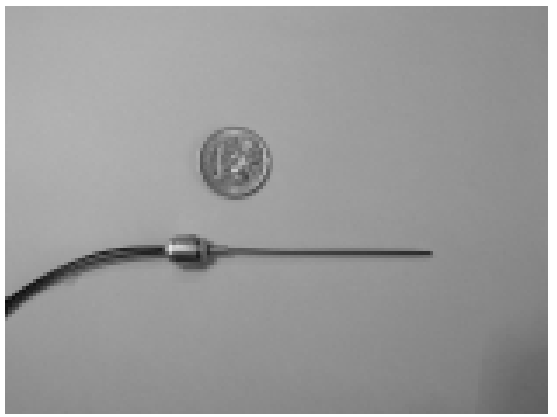

Fig. 2. Optical fibres vibrometer.

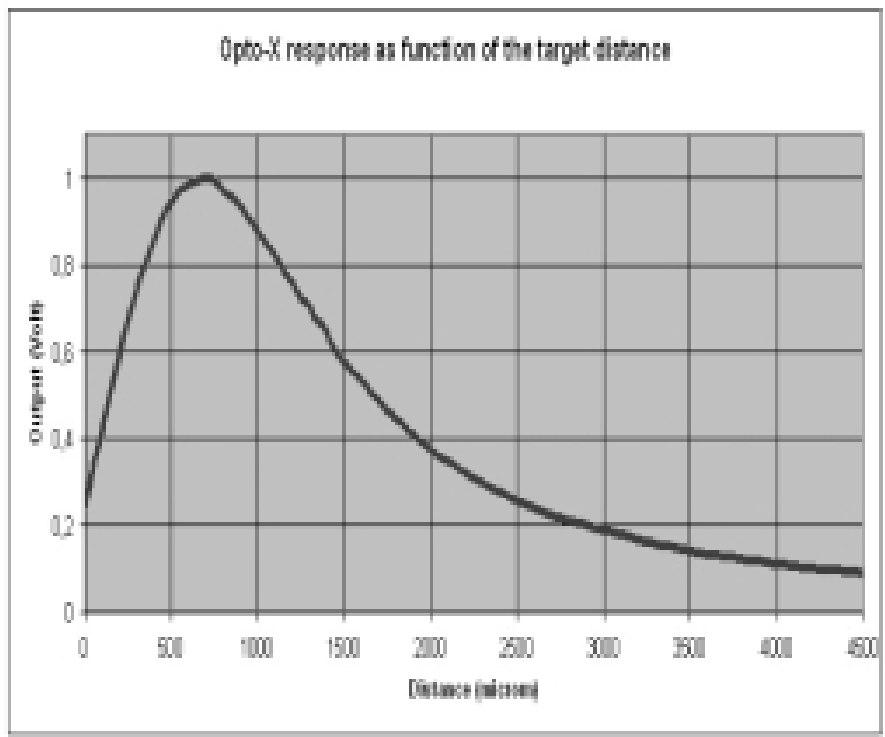

Fig. 3. Optical fibres vibrometer calibration curve.

applicability is far from general. Eventually the only effective solution is using contact-less sensors, that in no way modify the system's dynamic. In this direction, the laser based vibrometry is one of the preferred solutions, but it is a rather costly technology. In the following two contact-less sensors measurements will be compared: one is a commercially available device, based on hot wire anemometry, the other is an optical fibres sensors, developed 


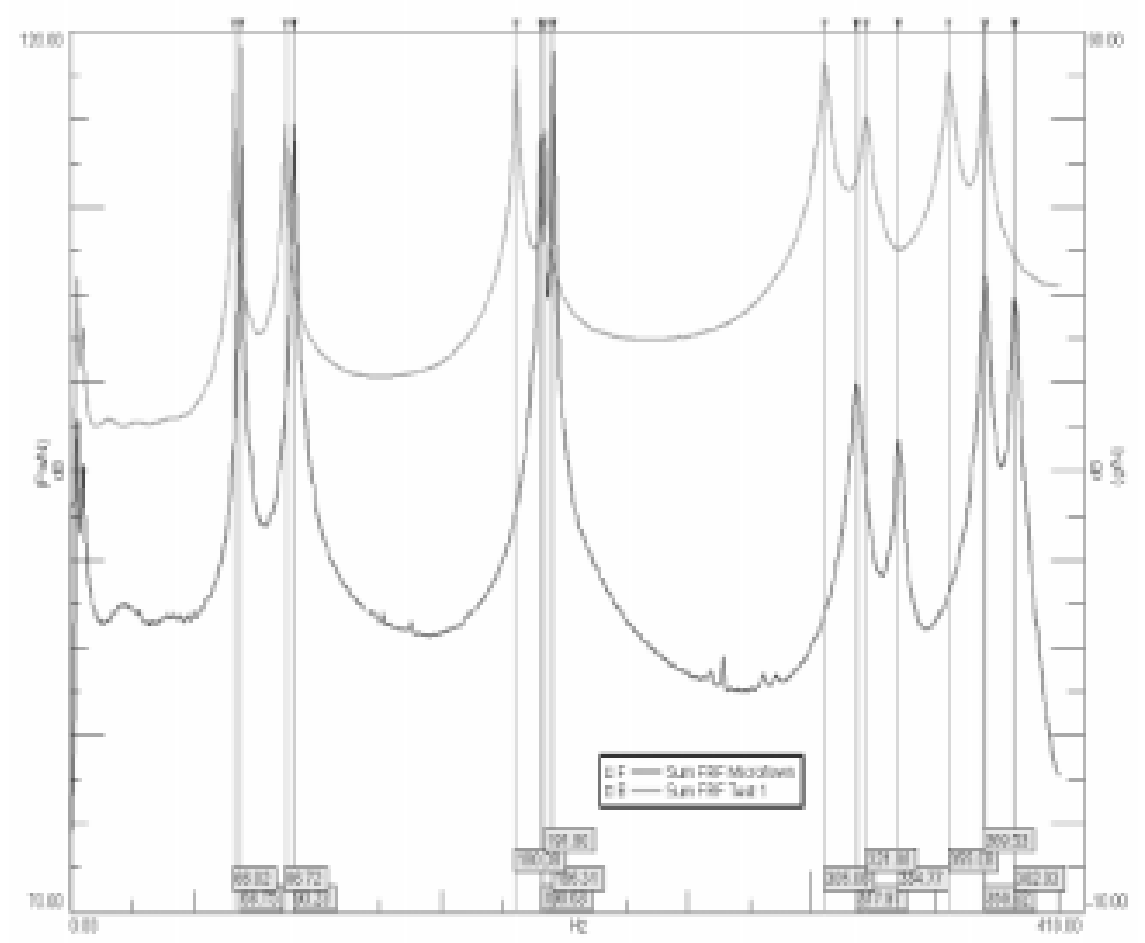

Fig. 4. FRF sum accelerometer (light-gray) vs microflown (dark-gray), plate test structure.

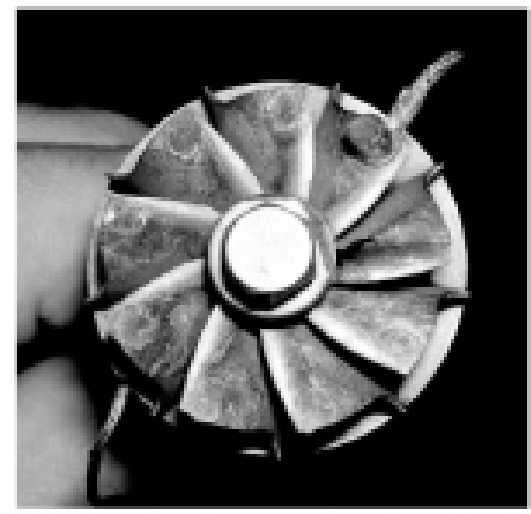

Fig. 5. The tested compressor.

by the authors. The results of the modal analysis performed on the experimental data acquired with the different transducers will be compared with the ones obtained with a standard accelerometer based acquisition chain.

\section{Microflown USP sensor}

The Microflown USP sensor consists of a miniaturized probe that allows measuring simultaneously, in one point, sound pressure and particle velocities in three directions. It's formed by three very small particle velocity sensors and a very small condenser microphone (Fig. 1). The particle velocity sensors are hot wire anemometers (red, blue, 
Table 1

The natural frequencies and the damping values for the accelerometer test

\begin{tabular}{cccccc}
\hline Mode & Frequency[kHz] & Damping $\%$ & Mode & Frequency[kHz] & Damping \% \\
\hline 1 & 21.927 & 0.09 & 9 & 24.323 & 0.05 \\
2 & 22.617 & 0.04 & 10 & 24.495 & 0.08 \\
3 & 22.687 & 0.04 & 11 & 24.613 & 0.98 \\
4 & 23.570 & 0.10 & 12 & 24.770 & 0.04 \\
5 & 23.698 & 0.23 & 13 & 24.892 & 0.07 \\
6 & 23.762 & 0.81 & 12 & 25.156 & 0.06 \\
7 & 23.921 & 0.04 & 15 & 25.200 & 0.08 \\
8 & 24.087 & 0.13 & - & - & - \\
\hline
\end{tabular}

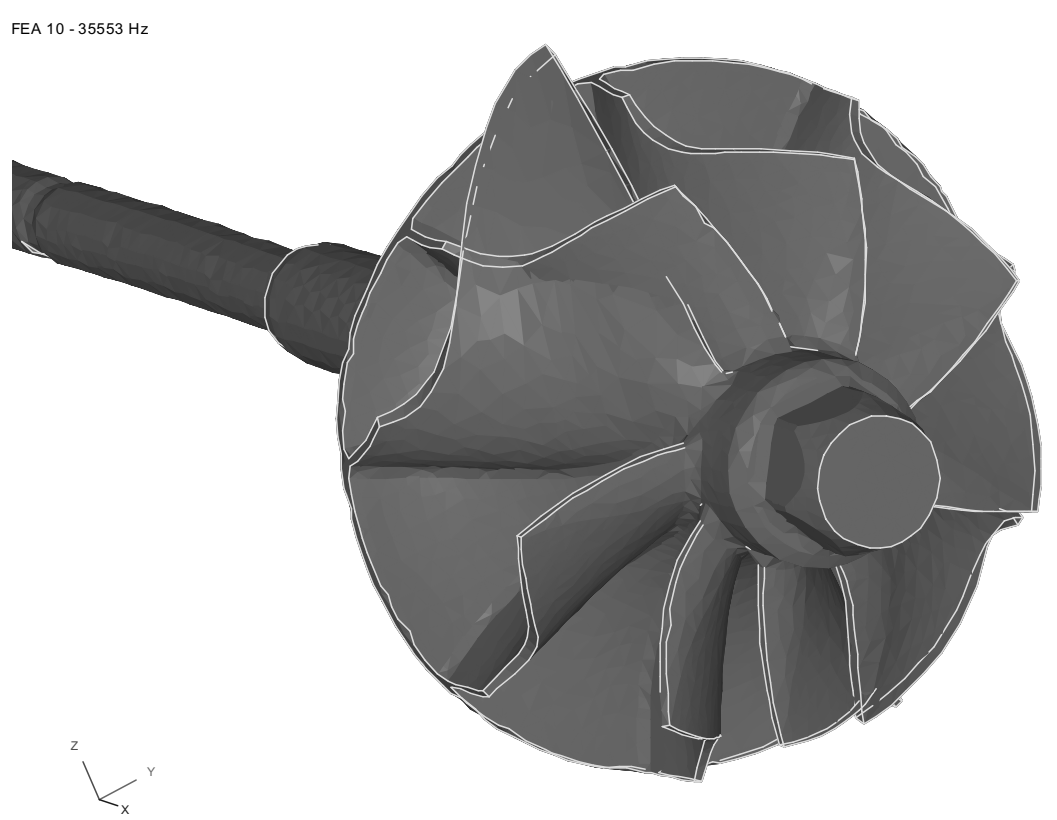

Fig. 6. A single blade mode, about $22 \mathrm{kHz}$.

green elements in the probe) consisting of two closely spaced wires heated up to about $300{ }^{\circ} \mathrm{C}$. The airflow generated by a vibrating surface, in the proximity of which the probe has to be placed during the measurements, causes a different cooling of the two wires. This differential cooling generates a difference in the electrical resistance between the wires, measured with a Weathsone bridge, that is proportional to the vibrating surface velocity. The probe, in the range $2-20 \mathrm{kHz}$, of has a sensitivity of about $20 \mathrm{mV} /(\mathrm{mm} / \mathrm{s})$ and $20 \mathrm{mV} / \mathrm{Pa}$ for the velocity and the pressure sensors respectively. To be calibrated it requires dedicated procedures and hardware. For further technical details, please refer directly to the producer website [7].

\section{Optical fibres vibrometer}

The optical fibres vibrometer consists of a sensor head and a processing unit. The head is formed by a set of optical fibres protected by a steel stylus. Of these fibres, some are connected to a light source (transmitting fibres) while the remaining (receiving fibres) are linked to a photo-detector. The stylus, Fig. 2, is placed in the proximity of the vibrating structure with its axis normal to the surface. The light, emitted by the transmitting fibres, is reflected by the moving surface and subsequently collected by the receiving fibres. The amount of light reaching the photo-detectors is proportional to the distance of the surface from the light source. The quantity of reflected light also depends from the characteristics of the vibrating surface, thus before the actual measurement, a calibration procedure has to be performed to define the sensor's calibration curve (Fig. 3). 
Table 2

The natural frequencies and the damping values for the Micro-flown test

\begin{tabular}{cccccc}
\hline Mode & Frequency[kHz] & Damping $\%$ & Mode & Frequency[kHz] & Damping \% \\
\hline 1 & 21.974 & 0.02 & 6 & 24.237 & 0.02 \\
2 & 22.876 & 0.02 & 7 & 24.598 & 0.02 \\
3 & 23.762 & 0.02 & 8 & 24.980 & 0.02 \\
4 & 24.035 & 0.02 & 9 & 25.252 & 0.02 \\
5 & 24.157 & 0.02 & - & - & - \\
\hline
\end{tabular}

Table 3

The natural frequencies and the damping values for the Optical Sensor test

\begin{tabular}{cccccc}
\hline Mode & Frequency[kHz] & Damping $\%$ & Mode & Frequency[kHz] & Damping \% \\
\hline 1 & 21.815 & 0.03 & 6 & 24.243 & 0.02 \\
2 & 22.874 & 0.03 & 7 & 24.612 & 0.03 \\
3 & 23.764 & 0.02 & 8 & 25.007 & 0.02 \\
4 & 24.051 & 0.02 & 9 & 25.251 & 0.02 \\
5 & 24.184 & 0.02 & - & - & - \\
\hline
\end{tabular}

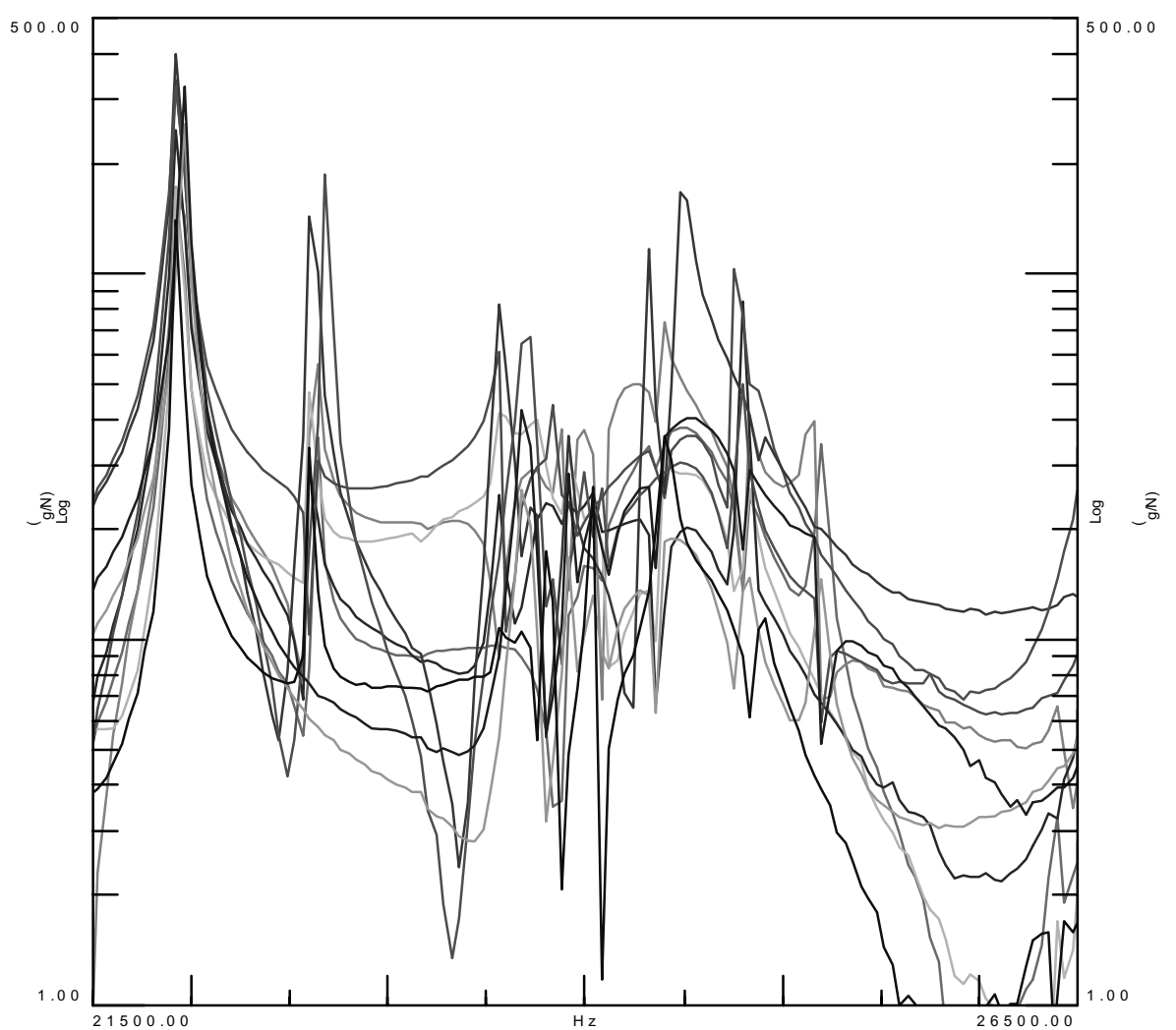

Fig. 7. Accelerometrs FRFs.

The sensitivity of the adopted combination of fibres is about $7 \mathrm{mV} / \mu \mathrm{m}$, in the band $0-300 \mathrm{kHz}$. The measuring range and measuring distance of the Optical Fibre Vibrometer greatly depend on the fibres dimensions, numbers and characteristics and can be tailored as function of the application. At the moment, sensors with measuring range from $100 \mu \mathrm{m}$ to $2 \mathrm{~cm}$ with a resolution from $3 \%$ to $0.05 \%$ are available. The processing unit elaborate the signal from the photo-detectors and delivers a signal that is proportional to the structure's displacement, velocity or acceleration. 


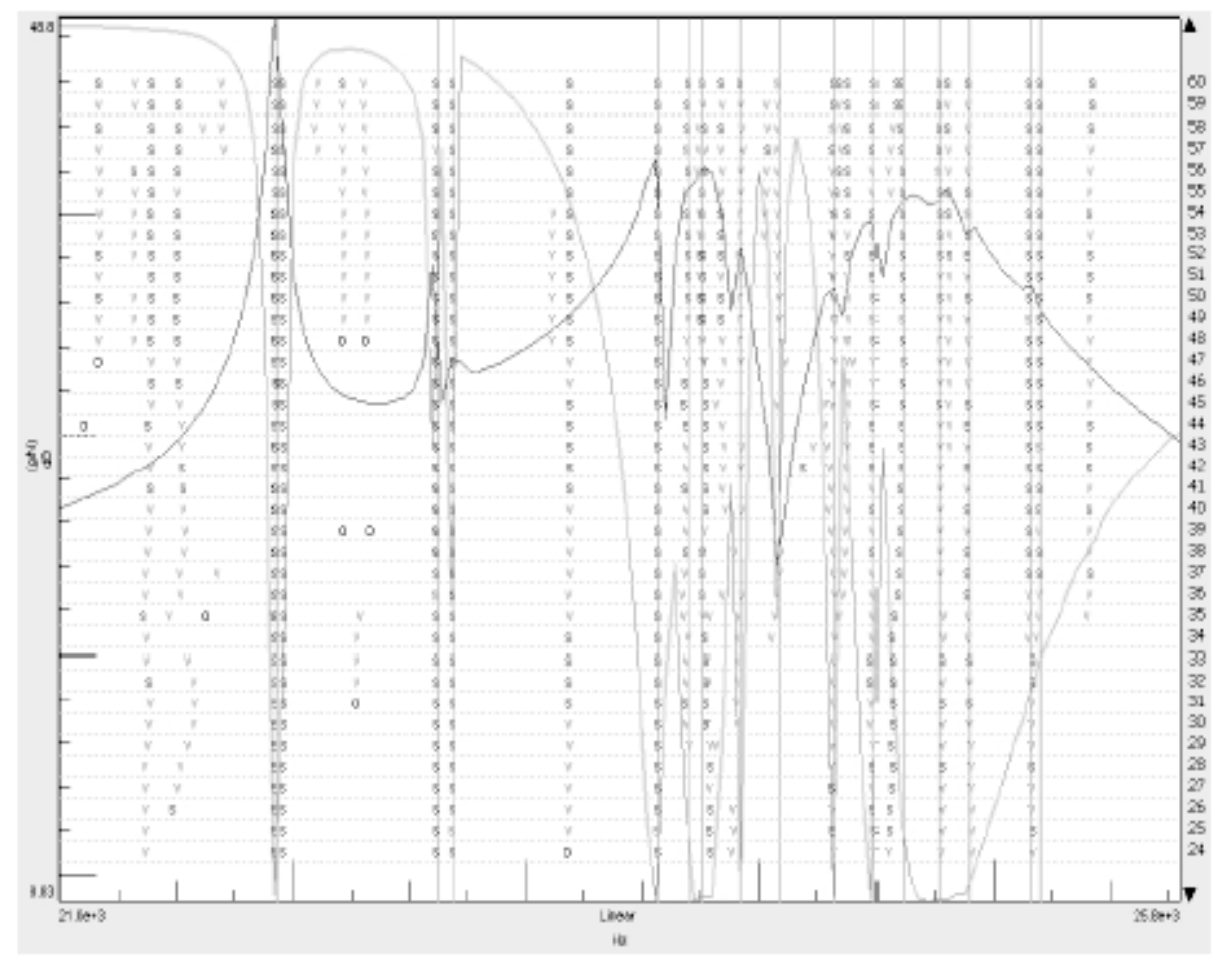

Fig. 8. The stabilization diagram accelerometers based test.

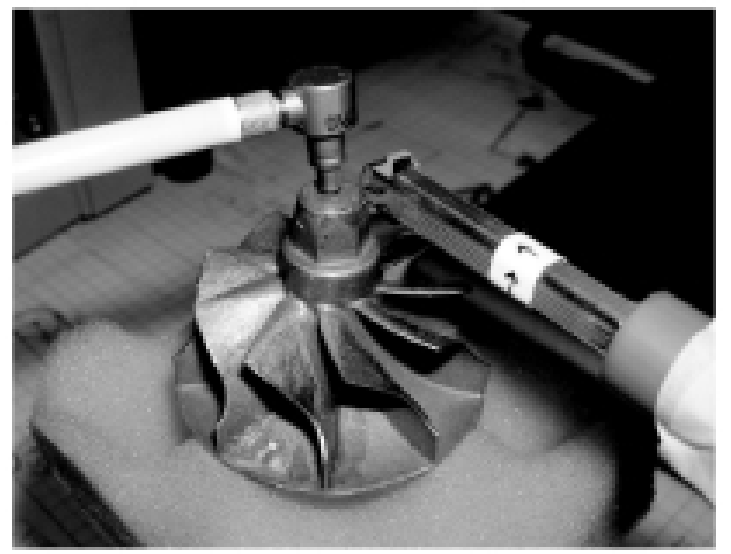

Fig. 9. The Microflown sensors and the micro hammer.

\section{Modal test on a small compressor}

\subsection{Accelerometer based testing}

The mass loading effect can be a problem already on a simple plate. Figure 4 shows the differences between the summed FRFs acquired with accelerometers or contact-less sensor [8]. In this case, a more complex structure is considered a radial compressor of a car turbocharger unit (Fig. 5). The test article has a disc diameter of $35 \mathrm{~mm}$, and the total weight is $56.6 \mathrm{~g}$. Such dimensions make the measurements a very difficult task, even if the very small 


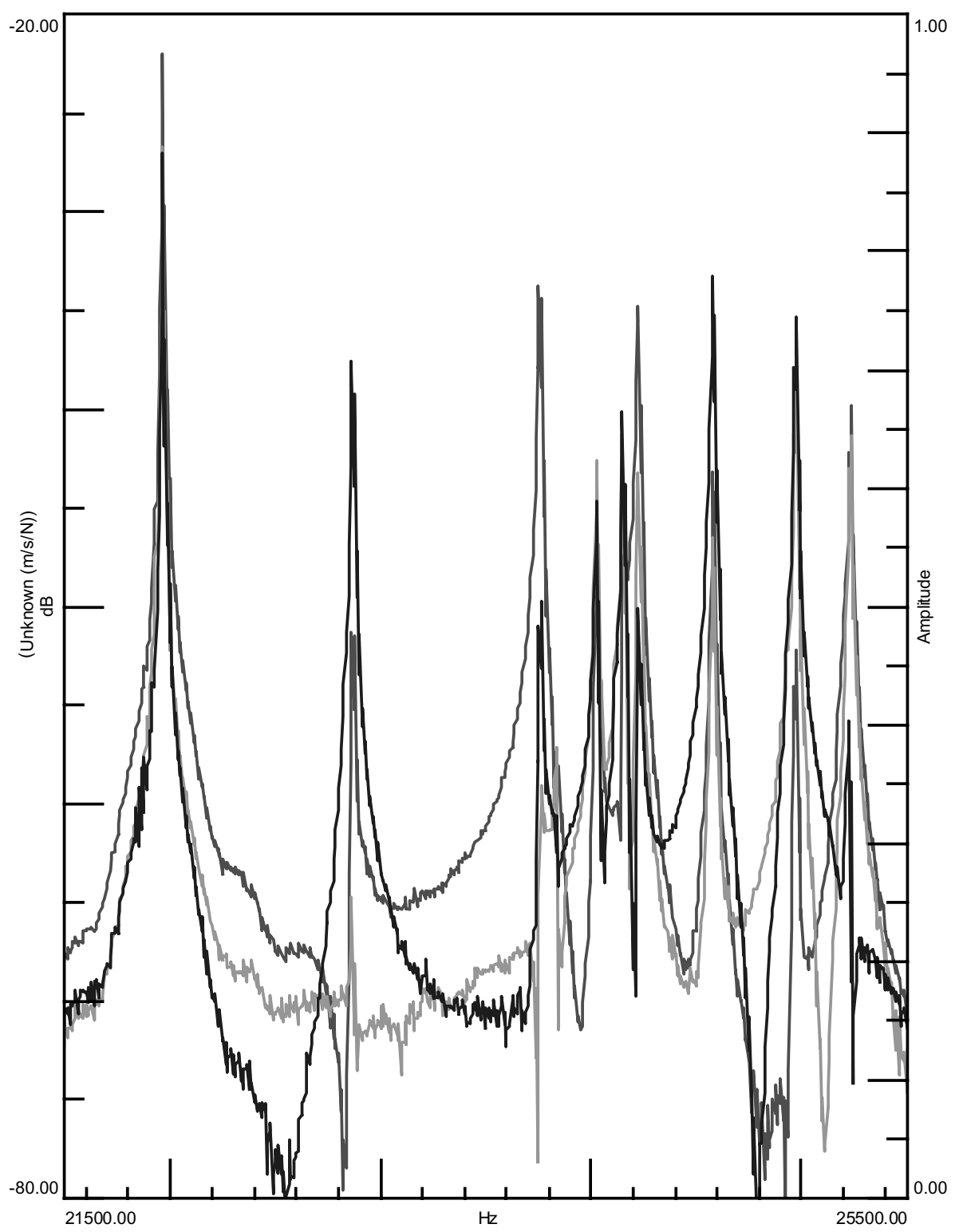

Fig. 10. Acquired FRFs blade 3, 6, 9 .

and light accelerometers are used. From the numerical simulation it's known that natural frequencies of the turbine blades lay well above the $20 \mathrm{kHz}$ (Fig. 6). This represents a further challenge for what concerns the excitation of the structure in the modal testing: it's very difficult to properly excite the structure with an impact hammer to such an high frequency range and obtain a sufficiently high level of signal for accelerometers. To minimize the mass loading effect problem the smallest available accelerometer $(0.15 \mathrm{~g})$ and a micro-hammer were used. The test technique was a standard modal testing with a multi-patch approach. A sensor was kept in a fixed position, for scaling and reference purposes, while another was placed, for each run, on a different blade. The excitation was always applied along the compressor axle, on the flat surface visible in the picture, to minimise the uncertainties linked to the excitation force direction.

Table 1 lists of modes identified in the band 20-26 kHZ. The identification algorithm (LMS PolyMAX) identifies fifteen modes, some of which with very limited frequencies differences, though only nine (one for blade) are expected. 


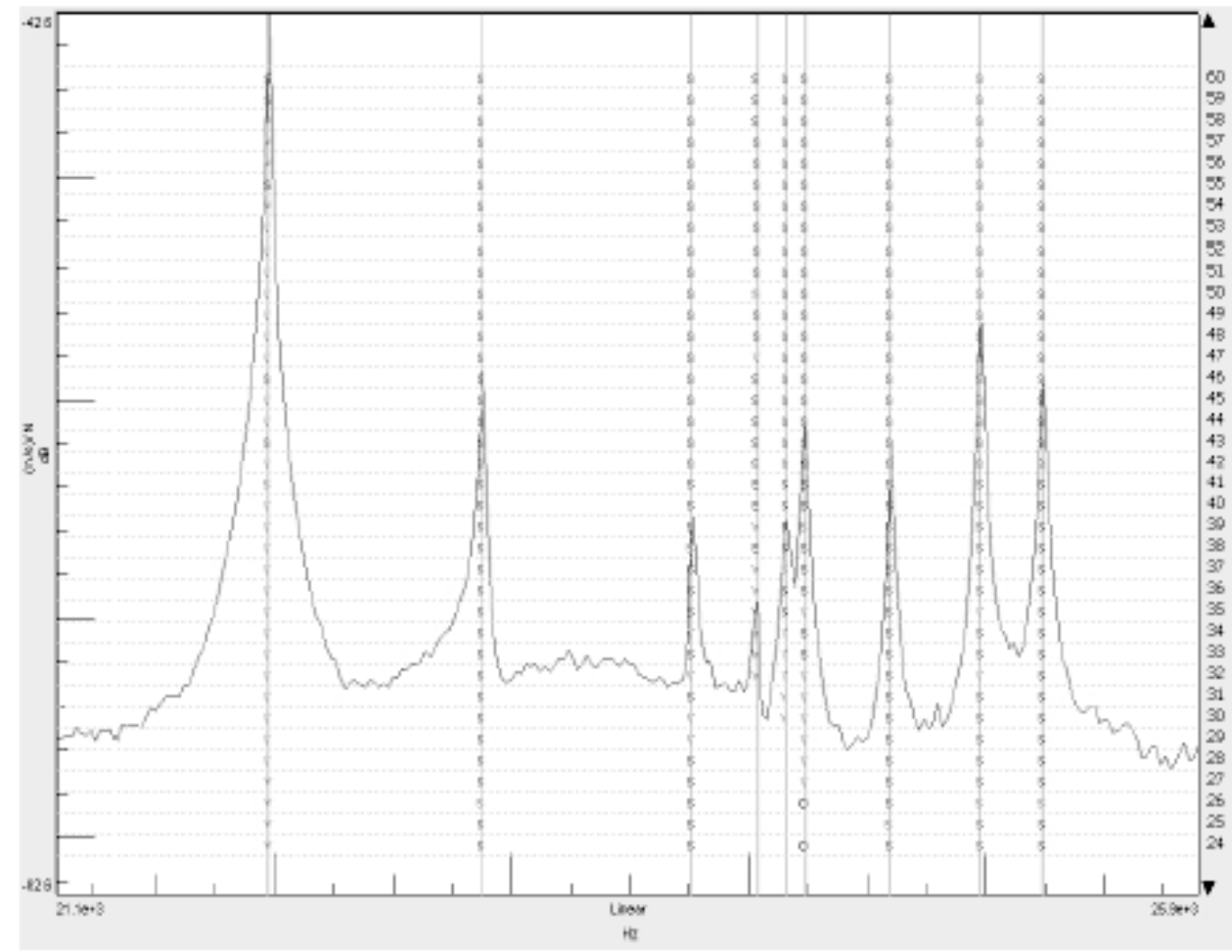

Fig. 11. The stabilization diagram Microflown based test.

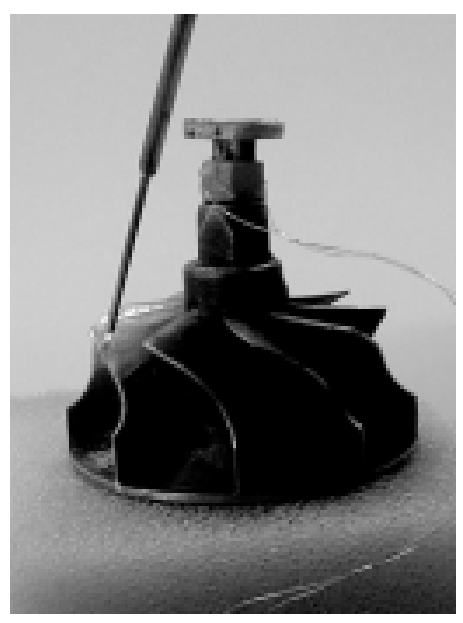

Fig. 12. Optical sensor and the micro shaker.

The extra poles are the results of the mass loading and the subsequent data inconsistency between the measurement runs. Also the damping values exhibit some strange very high values, due to the overlapping of slightly shifted resonance peaks (see Figs 7-8) and the subsequent peaks broadening. Even the selected powerful identification algorithm, does not help much discriminating between real and virtual modes and their animation shows very similar and quite complex deformation shapes. 


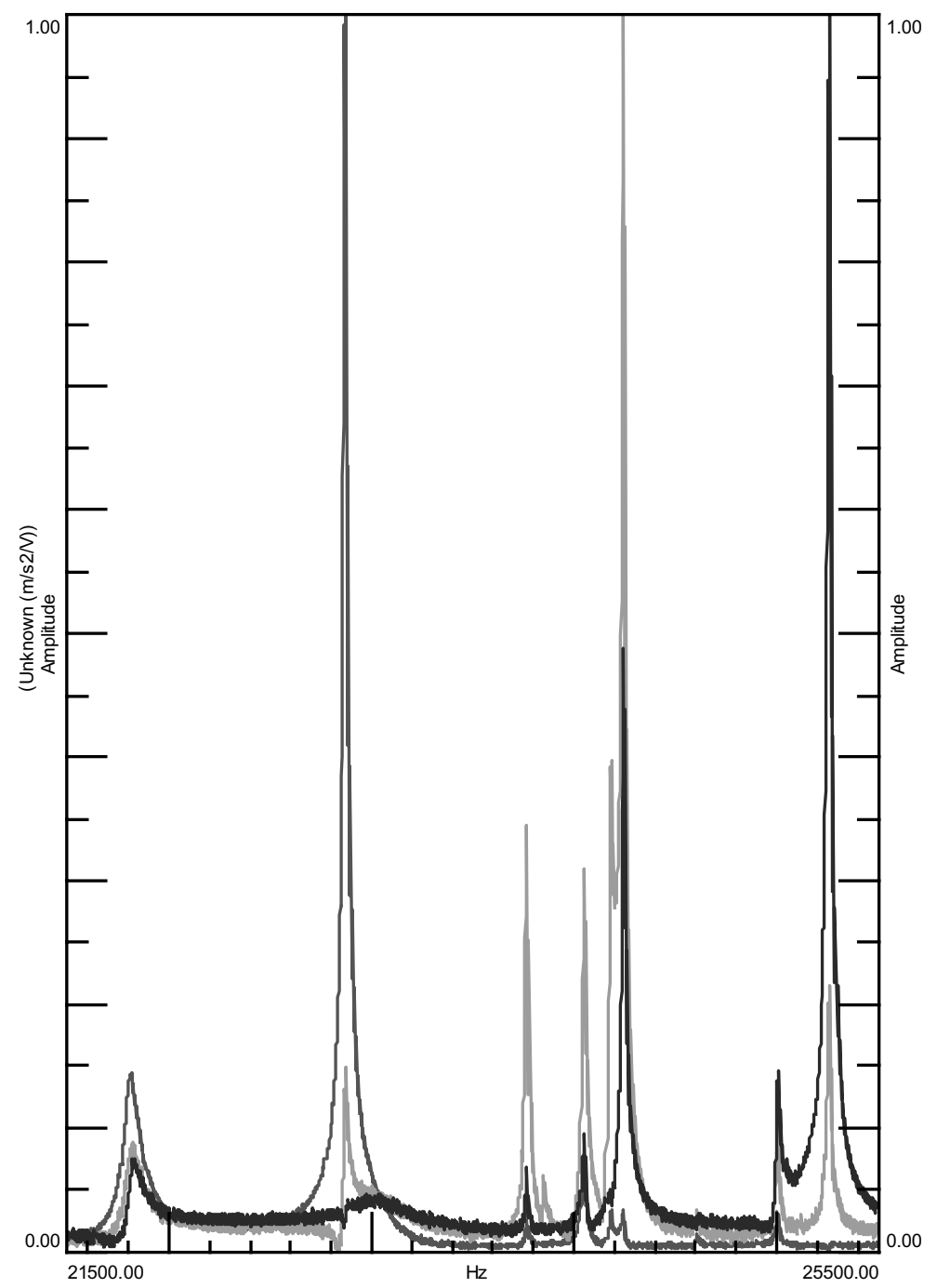

Fig. 13. Acquired FRFs blade 3, 6, 9.

\subsection{Micro-flown based testing}

Similarly to the accelerometers based tests, the Microflown acquisition was conducted in multiple runs: one for each blade plus one for the driving point FRF (Fig. 9). In these case, the FRFs were obtained without contact and the consistency of the database is evident when looking at the peaks of the acquired FRFs.

Figure 10 shows the data measured for blade 3, 6 and 9 while Fig. 11 shows the stabilization diagram obtained with the PolyMAX identification tool. In this case it's evident the cleanliness of the diagram and the lack of multiple modes. In the frequency band of interest only the foreseen 9 modes appear. Table 2 lists the identified modes frequencies and damping values. These latter are all clustered around a single value, specifically 0.02 , compatible for such an all-metal little structure.

\subsection{Optical fibre vibrometer based testing}

For the optical sensor measurement, multiple runs were performed as well. For the chosen fibre sizes and the nature of the light source, in order to work in one of the two linear range of the sensor, the distance between the 


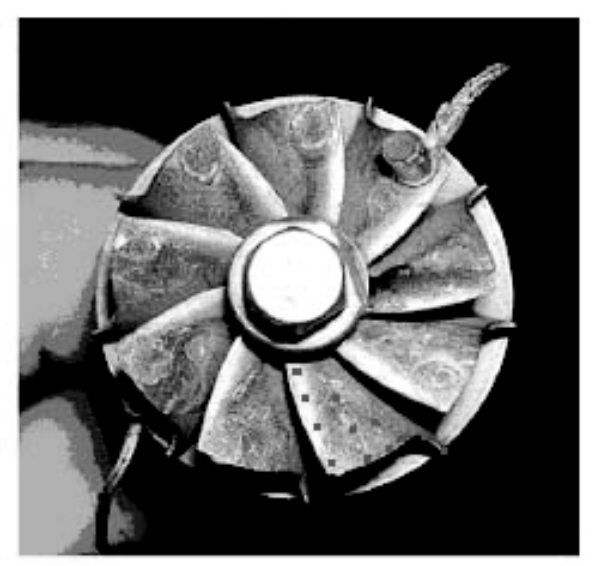

Fig. 14. Blade's measure points.

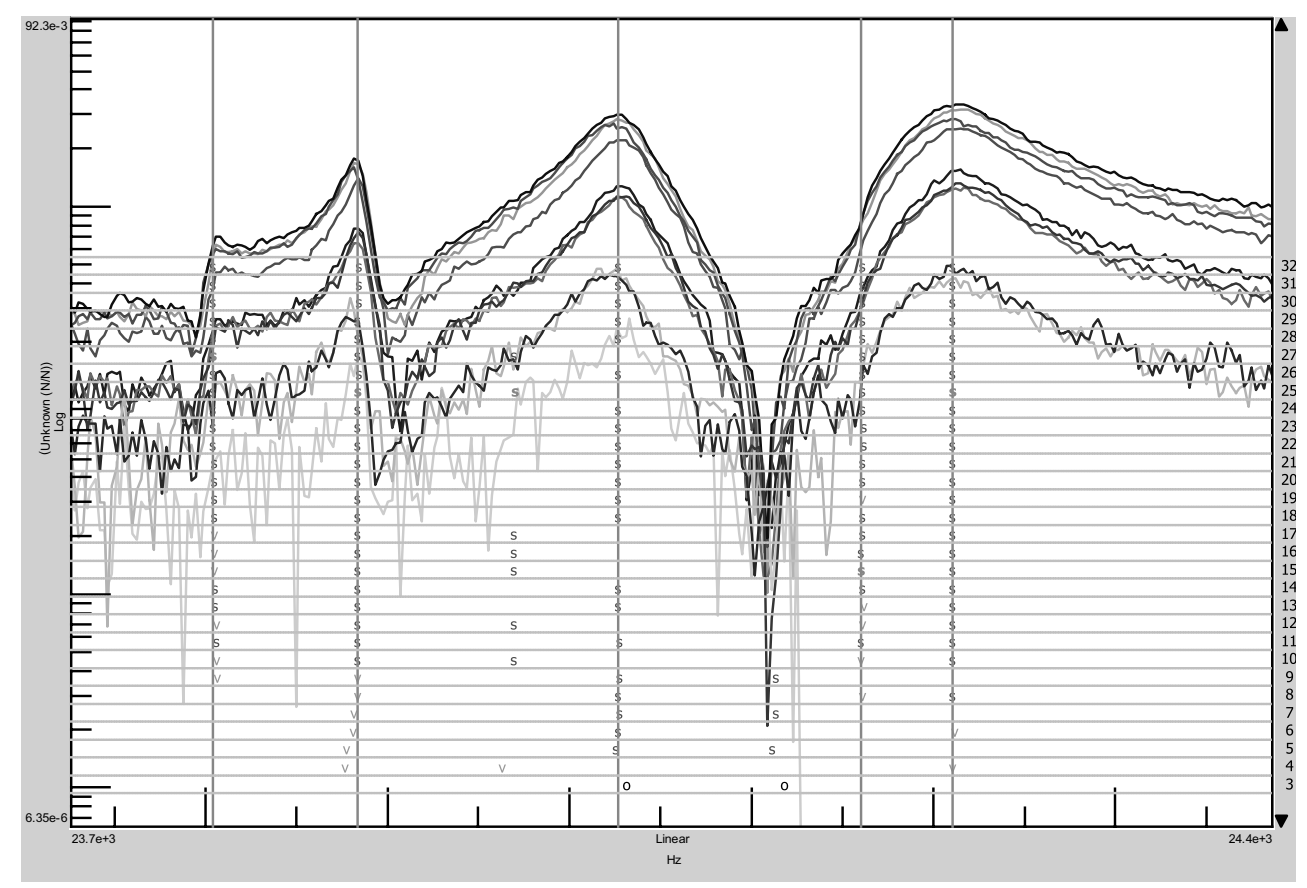

Fig. 15. Stabilisation diagram for the blade mapping measurement.

stylus tip and the object is about $1 \mathrm{~mm}$. This set up assures for the highest signal sensibility, but it also requires that the applied structure excitation doesn't displace excessively the test structure, altering the distance sensor-test object. For this reason the impact hammer excitation was substituted with a micro inertial shaker. This device is in the early development stage at the LVA. The dynamic effect of the shaker's added mass can be accounted for, both the injected force and the value of the added mass are known. This solution allows to excite the structure in an easy and controllable way from about $5 \mathrm{~Hz}$ to $50 \mathrm{kHz}$. Figure 12 shows the optical sensor and the prototype of the inertial shaker, while Fig. 13 shows the typical measured tip blade responses.

Due to the contact-less nature of this sensors, the measurements coming from the different runs look very consistent to each other and the stabilisation diagram is very similar to the one obtained with the Microflown sensor, Fig. 11. Table 3 shows the natural frequencies and the damping values identified with the optical sensor that are very close 


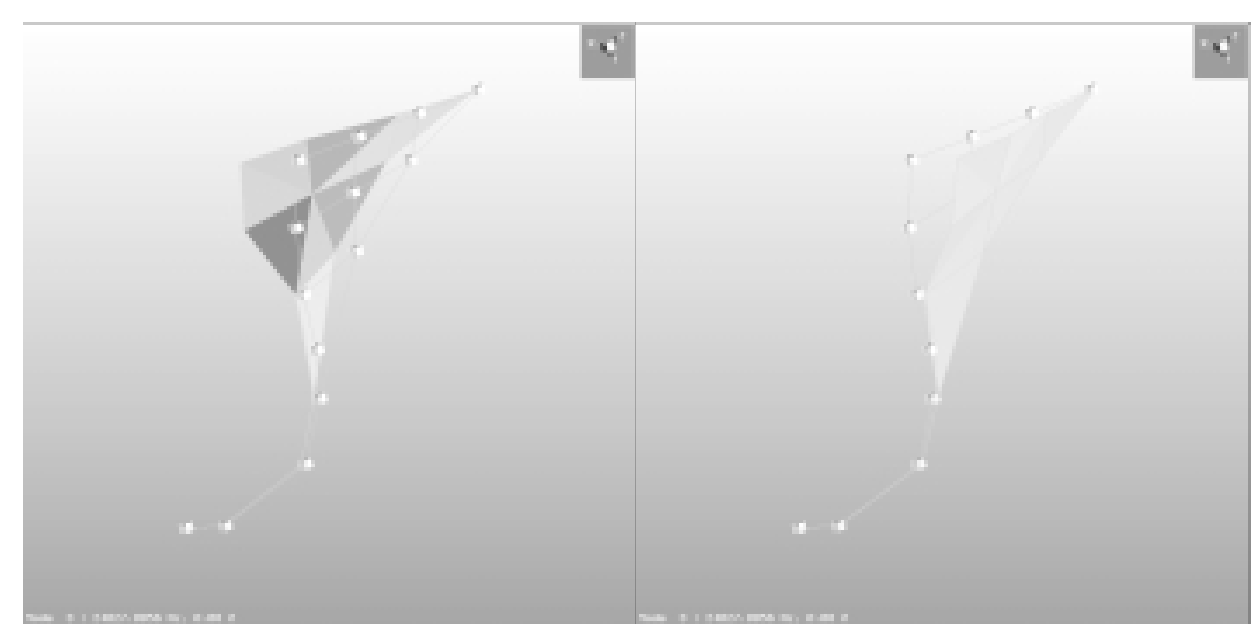

Fig. 16. Blade's mode shape at $24050 \mathrm{~Hz}$.
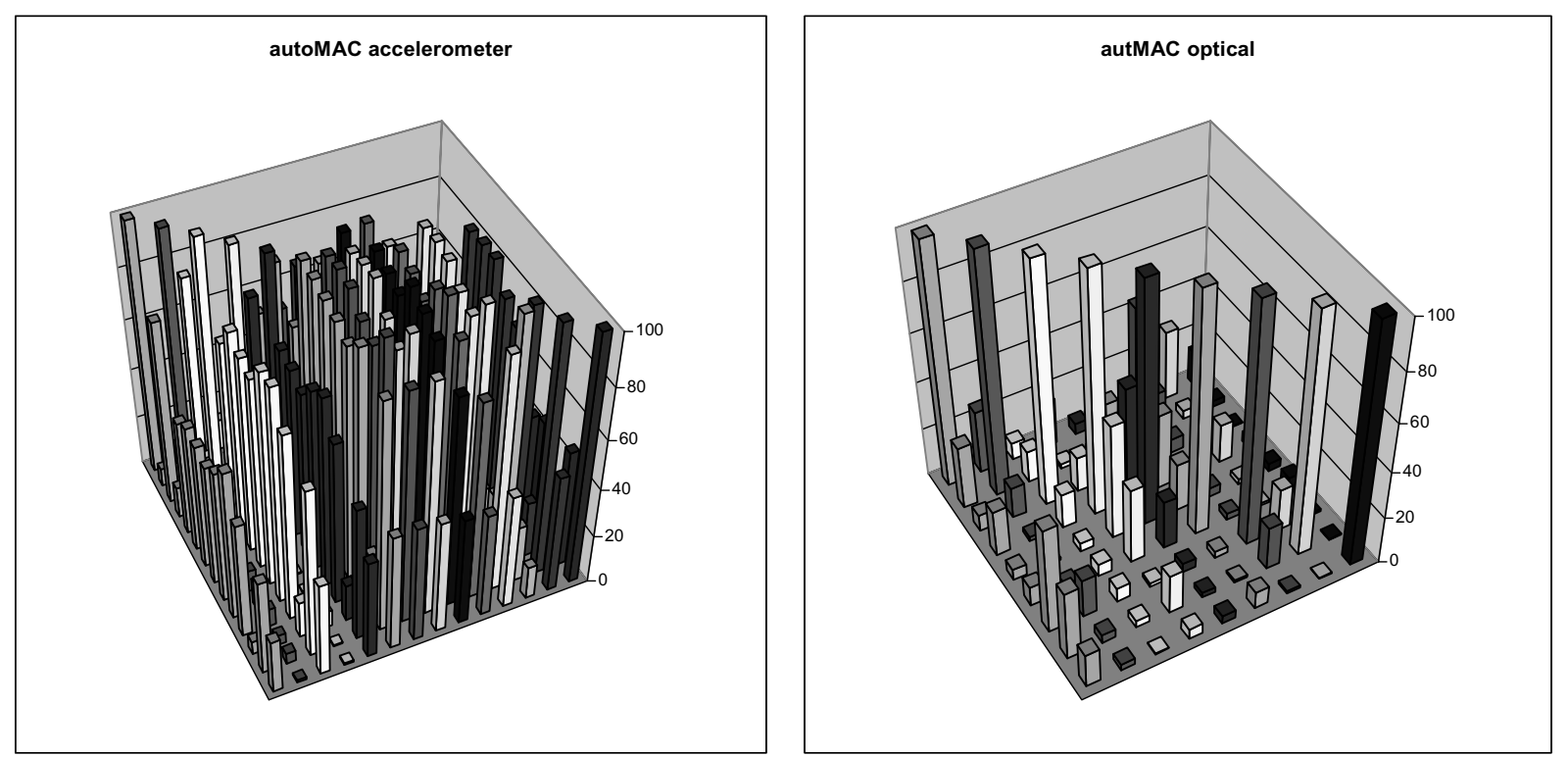

Fig. 17. AutoMac Matrices (L) accelerometer based measure, (R) optical based measure.

to the ones obtained with the other contact-less sensor.

\subsection{Single blade vibration mapping}

Due to the small measuring spot, the optical sensor can be used to map the vibration of small part of the structure. In the specific case, a single blade has been measured, acquiring the data on 14 evenly spaced points some of which are displayed in Fig. 14. It's worth remembering that the size of the leading edge of the blade is about $10 \mathrm{~mm}$ and the distance between the measuring points is about $2 \mathrm{~mm}$. The FRFs have been acquired in a range $22000-25000 \mathrm{~Hz}$ with a resolution of $1 \mathrm{~Hz}$ using as exciter the micro-inertial shaker. The data and the stabilisation diagram are reported in Fig. 15. Also in this case the PoliMAX algorithm has been used to identify poles and modes. Figure 16 shows the deflection of one blade at $24050 \mathrm{~Hz}$. 


\section{Conclusions}

This paper describes three alternative methods to acquire the data necessary to conduct a modal testing on a lightweight structure. The advantage of the contact-less sensors becomes evident when comparing the FRFs, acquired with the different techniques (Figs 7, 9,12) or evaluating the results of the identification phase with the auto-MAC matrix (Fig. 17). Here, it can be seen that while for the accelerometer based test, the modes present high level of correlation (high off diagonal MAC values) for the contact-less cases, the different modes are clearly identified (low off diagonal MAC values). Further evaluation will be carried out in the future, adopting the same excitation for the different sensors. Velocity and acceleration outputs will be added to the optical sensor for direct and immediate comparisons with the Microflown and the accelerometers data. The reported activities focus on very small and lightweight structure, but advantages are expected also in the identification of non linearities taking advantage of the high sensitivity of the optical sensor and the possibility to finely tune excitation with the micro inertial shaker.

\section{References}

[1] Van Der Auweraer, H. Leurs and W. Mas P: Hermans, Modal parameter estimation from inconsistent data sets, Proceedings 18th IMAC Sant. Antonio, 2000, 763-771.

[2] A. Vecchio, L. Valent and L. Bregant, Impact of Test Data Uncertainties on Modal Models extracted from Multi-Patch Vibrations Test, Proceedings 1st INCE Symposium, Le Mans, 2005.

[3] N. Baldanzini and M. Pierini, An assessment of transducers mass loading effects on the parameters of an experimental statistical energy analysis (SEA) model, Mechanical Systems and Signal processing 16 (2002), 885-903.

[4] M.R. Ashory, Correction of mass-loading effects of transducers and suspension effects in modal testing, Proceedings of the 16th ISMA, Leuven, 1998, 815-828.

[5] O. Dossing, Prediction of transducer mass-loading effects and identification of dynamic mass, Proceedings of the 9th IMAC, Firenze, 1991, 306-311.

[6] O. Cakar and K.Y. Sanliturk, Elimination of transducer mass loading effects from frequency response function, Mechanical system and Signal Processing 19 (2005), 87-104.

[7] http://www.microflown.com.

[8] L. Valent, A. Vecchio and L. Bregant, Particle velocity sensors for modal testing of light weight structures, Proceeding of SEM Annual Conference, Saint Louis, 2007. 

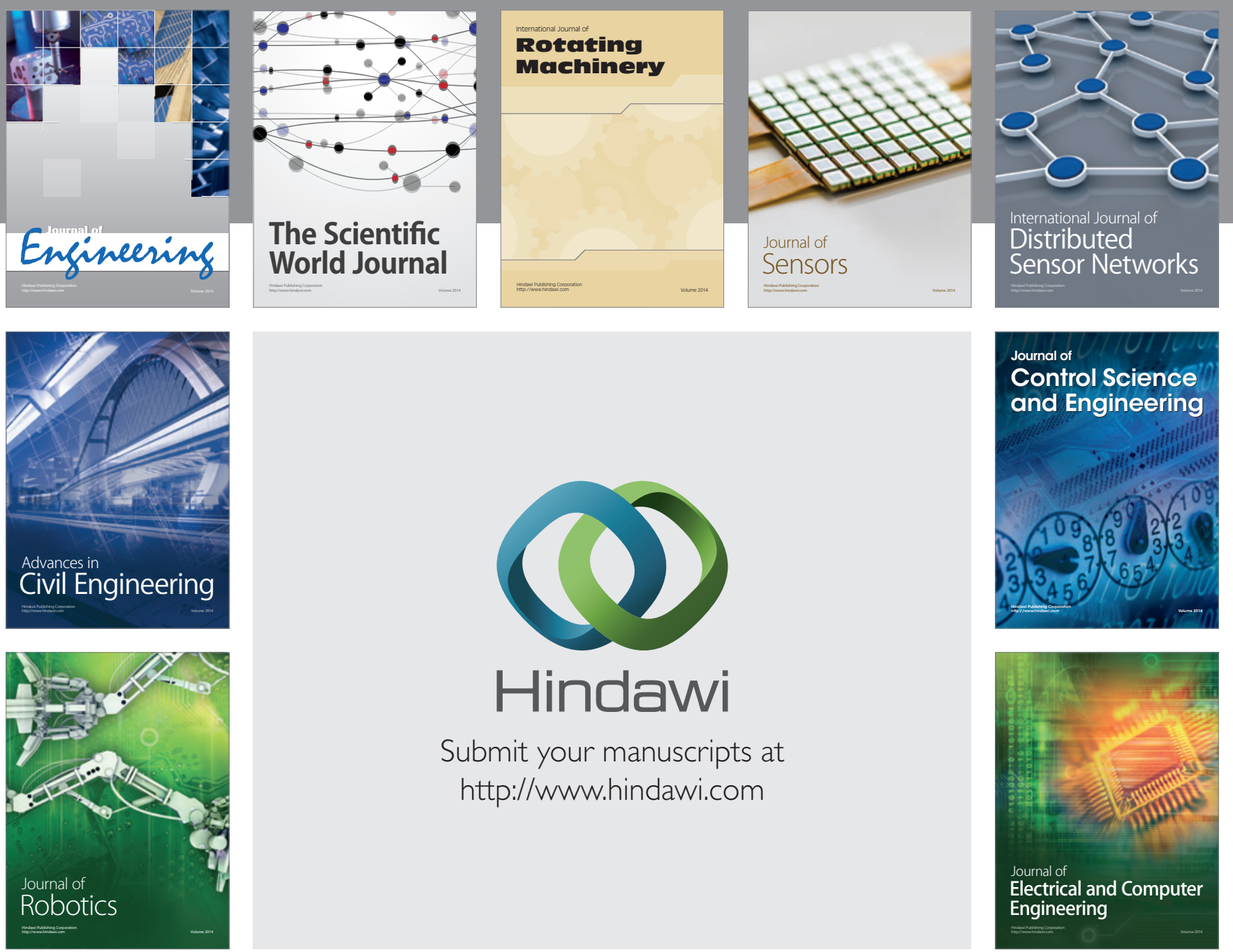

Submit your manuscripts at

http://www.hindawi.com
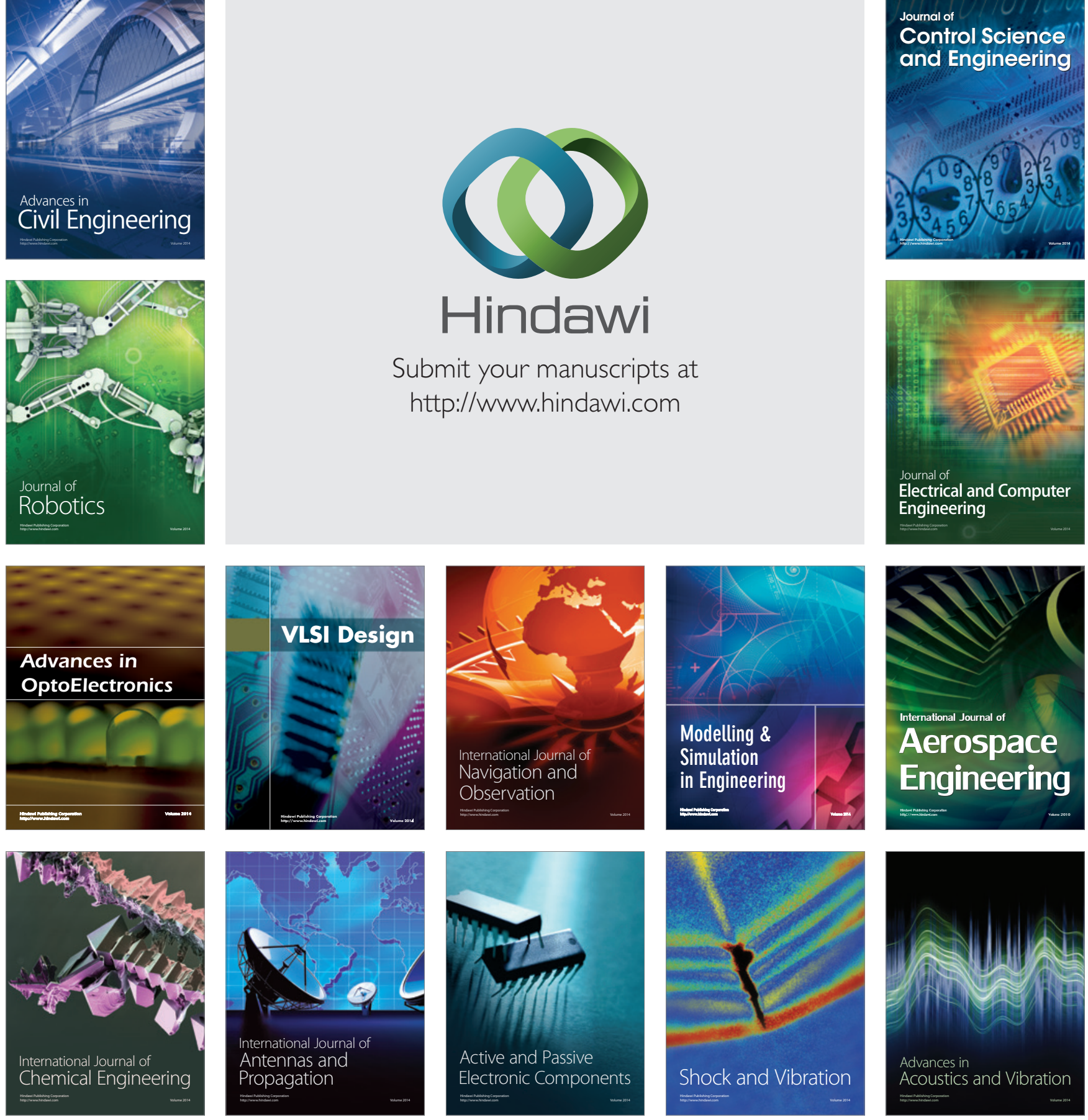